\title{
Tabaquismo en adolescentes de un rango de 10 a 18 años
}

\author{
Smoking in teenagers between 10 and 18 years old \\ María L. Sánchez-Padilla ${ }^{a}$,Viridiana Ramírez-Gutiérrez ${ }^{b}$, José Arias-Rico $^{c}$, \\ Rosario Barrera-Gálvez ${ }^{d}$, Reyna C. Jiménez-Sánchez ${ }^{e}$, Margarita Lazcano-Ortiz ${ }^{f}$, \\ Karina Chávez-Rubio ${ }^{g}$, Abigail Martínez Alamilla ${ }^{h}$
}

\begin{abstract}
:
Tobacco is an addictive substance, mainly due to its active component, nicotine, which acts on the central nervous system. The smoker suffers a physical and psychological dependence that generates a withdrawal syndrome, called smoking. Nicotine generates addiction, but it has antidepressant and symptomatic relief effects of anxiety. Most adolescents are aware that tobacco use is one of the biggest causes of death. However, this does not deter them from trying tobacco products. Trying tobacco only once puts you at risk of becoming addicted to nicotine.
\end{abstract}

\section{Keywords:}

Tobacco, teenagers

\section{Resumen:}

El tabaco es una sustancia adictiva, debido principalmente a su componente activo, la nicotina, que actúa sobre el sistema nervioso. El fumador sufre una dependencia física y psicológica que genera un síndrome de abstinencia, denominado tabaquismo. La nicotina genera adicción, pero tiene efectos antidepresivos y de alivio sintomático de la ansiedad. La mayoría de los adolescentes son conscientes de que el consumo de tabaco es una de las mayores causas de muerte. No obstante, esto no los disuade de probar productos del tabaco. Probar tabaco tan solo una vez los pone en riesgo de volverse adictos a la nicotina. El presente ensayo da a conocer la problemática que genera el que los adolescentes sean adictos al tabaco.

\section{Palabras Clave:}

Tabaco, adolescentes

\footnotetext{
${ }^{a}$ Universidad Autónoma del Estado de Hidalgo, Instituto de Ciencias de la Salud , Email: marialuisasanchezpadilla@yahoo.com

b Universidad Autónoma del Estado de Hidalgo, Instituto de Ciencias de la Salud, Email: viri_gutierrez16@ hotmail.com

c Universidad Autónoma del Estado de Hidalgo, Instituto de Ciencias de la Salud, Email: josearias.rico@ hotmail.com

d Universidad Autónoma del Estado de Hidalgo, Instituto de Ciencias de la Salud, Email: Rosario_barreara@uaeh.edu.mx

e Universidad Autónoma del Estado de Hidalgo, Instituto de Ciencias de la Salud, Email: cristyji@ hotmail.com

${ }^{\mathrm{f}}$ Universidad Autónoma del Estado de Hidalgo, Instituto de Ciencias de la Salud, Email: magos1000@ @otmail.com

g Universidad Autónoma del Estado de Hidalgo, Instituto de Ciencias de la Salud, Email: kary070895@ @otmail.com

${ }^{\mathrm{f}}$ Universidad Autónoma del Estado de Hidalgo, Instituto de Ciencias de la Salud, Email: abigailmartinez5808@ gmail.com
} 


\section{Desarrollo}

La OMS clasifica al tabaquismo como una enfermedad crónica no transmisible y adictiva. Es una adicción legal, que se integra social y culturalmente al comportamiento humano. Aunque se ha avanzado en la educación sobre su nocividad y su relación con la enfermedad y la muerte, estudios realizados en Cuba encuentran que los fumadores tienen conocimiento sobre el tabaquismo, pero no percepción de sus riesgos. Ejemplo de esto es el hecho de que está presente en los estudiantes y profesionales de la salud, que han aprendido a identificar el riesgo de eventos no saludables en los pacientes, y no siempre reconocen su propio riesgo de enfermar o morir.

La exposición al humo del tabaco es un problema grave en las etapas del desarrollo, y casi la mitad de infantes y adolescentes respiran aire contaminado en lugares públicos. Cada día empiezan a fumar entre 82000 y 99000 jóvenes, muchos de ellos menores de 10 años.

El humo del tabaco contiene más de 4.000 productos químicos nocivos, como alquitrán, monóxido de carbono, acetaldehído y nitrosaminas, entre otros. Al inhalar el humo, se consume de 1 a $2 \mathrm{mg}$ de nicotina por cigarrillo, que llega al torrente sanguíneo en $10 \mathrm{~s}$ y penetra al cerebro. La nicotina aumenta el potencial para su abuso al activar los circuitos que regulan los centros del placer conocidos como circuitos de gratificación. El neurotransmisor dopamina aumenta, lo mismo que con otras drogas de abuso, y es la causa de las sensaciones placenteras que experimentan los que fuman. Los efectos agudos de la nicotina se disipan rápidamente, por lo que los fumadores continúan dosificándosela repetidamente para mantener la sensación placentera, y evitar el síndrome de abstinencia.

Entre los problemas de salud causados por el tabaco se incluyen: deterioro dental, daños metabólicos, tos frecuente, aumento de flema, disminución del buen estado físico, problemas respiratorios, irritabilidad, ansiedad, deseos vehementes por la droga, déficit cognitivo, trastornos del sueño y del apetito, impaciencia e intranquilidad, que comienzan pocas horas después de haber dejado de fumar, obligando a la persona a volver a hacerlo

- El $90 \%$ de las personas que consumen tabaco a diario comienzan a hacerlo a los 18 años de edad.

- El $25 \%$ de los alumnos de escuela secundaria manifestaron consumir actualmente un producto del tabaco, incluyendo un $13 \%$ que manifestó el consumo actual de dos 0 más productos del tabaco.

- Entre los tipos de productos del tabaco consumidos por alumnos de escuela secundaria se incluyen: cigarrillos electrónicos, narguiles, cigarrillos, cigarros (cigarros pequeños 0 finos como Swisher Sweets o Black and Mild), tabaco sin humo, pipas, snus (tabaco de consumo oral), bidis (cigarrillos de tabaco enrollado) y tabaco soluble.

\section{Signos que pueden delatar el hábito de fumar son:}

- Tos

- Irritación de la garganta

- Ronquera

- Mal aliento

- Un rendimiento inferior en los deportes

- Mayor susceptibilidad a los resfriados

- Dificultades respiratorias

* Los factores que pueden influir en el consumo de tabaco son:

- Consumo de productos de tabaco por parte de amigos o familiares.

- Falta de apoyo o involucramiento de los padres.

- Fácil acceso, disponibilidad y precio accesible de los productos del tabaco.

- Bajo rendimiento académico.

- Baja autoestima.

- Exposición a publicidad del tabaco (películas, televisión o videojuegos).

\section{* Consejos para evitar que los adolescentes consuman productos del tabaco}

- Dé el ejemplo a sus hijos. Los hijos de fumadores actuales o ex fumadores enfrentan un mayor riesgo de convertirse en fumadores.

- Si fuma, intente dejar de hacerlo. Consiga el apoyo de su familia. Ver lo difícil que le resulta dejar de 
- fumar podría ser suficiente para evitar que sus hijos comiencen a hacerlo. Designe su casa y su automóvil como zonas libres de humo.

- Cuente a sus hijos sobre los efectos secundarios del tabaquismo. Fumar perjudica la capacidad atlética, provoca arrugas, da mal aliento, mancha los dientes y cuesta mucho dinero.

- Si los adolescentes empiezan a fumar, animar a dejar de hacerlo. Al dejar de fumar, las personas añaden años a su vida.

- Informar de temas delicados de un modo que no haga sentir a sus hijos temor al castigo o a ser juzgados.

- Anime a los niños a implicarse en actividades en las que se prohíbe fumar, como los deportes.

- Establecer programas educativos permanentes a edades tempranas, entre 6 y 10 años, para prevenir el consumo del tabaco.

\section{CONCLUSIÓN}

Llegamos a la conclusión de que para ayudar a evitar que los adolescentes consuman tabaco, incluyendo la restricción de la publicidad, el aumento de precios de los productos y el aumento de la edad legal para comprar productos del tabaco.

Mantener una buena comunicación, padres e hijos, eso ayudara a darse cuenta del comportamiento de los adolescente y así mismo saber quiénes son sus amistades.

Establecer programas intersectoriales de salud y educación que tengan como propósito fundamental la prevención del consumo de tabaco, y así mismo fomentar platicas con los padres de familia para que sepan la importancia del consumo de tabaco.

\section{Referencias}

[1] Henriquez, 1. g. (2002). consumo de tabaco en adolescentes: factores . Ciencia y enfermería.

[2] Lando, H. A. (2010). El tabaco es un problema que afecta a los niños en el mundo entero. Organización Mundial de la Salud.

[3] Monteverde, D. H. (2001). Programa de Acción. Secretaria de salud.

[4] Ramírez, M. d. (2017). Tabaquismo en la infancia y la adolescencia: una adicción, un reto. Revista, Cubana Pediatrica .

[5] Steven Dowshen, M. (2013). Los niños y el tabaco. KidsHealth / para Padres / Los niños y el tabaco . 